\title{
Rook version of colored partition algebras
}

\author{
A. Joseph Kennedy • G. Muniasamy
}

Received: 23 October 2011 / Revised: 7 March 2012 / Accepted: 12 March 2012 /

Published online: 5 April 2012

(C) The Author(s) 2012. This article is published with open access at SpringerLink.com

Abstract We study the rook version of the colored partition algebras $P_{k}(n, G)$ and $\widehat{P}_{k}(n, G)$ and we obtain the corresponding Schur-Weyl dualities.

Keywords Partition algebra $\cdot$ Centralizer algebra $\cdot$ Direct product $\cdot$ Wreath product . Symmetric group

Mathematics Subject Classification $\quad 16$ S20 $\cdot 16$ S50 16 S 99

\section{Introduction}

There are various deformations of semigroup algebras arising from the generalizations of the classical Schur-Weyl duality. The partition algebras $P_{k}(x)$ have been studied independently by Martin and Jones, as a generalization of the Temperley-Lieb algebras and the Potts model in statistical mechanics (see [7]). In 1993, Jones considered $P_{k}(n)$, as the centralizer algebra of the symmetric group $S_{n}$ on $V^{\otimes k}$ (see [5]).

The Class (or Ramified) partition algebra $P_{k}(m, n)$ has been introduced in [8] also in [6] by Kennedy, and has been realized as the centralizer algebra of the wreath product $S_{m} 2 S_{n}$ acting on the tensor space $W^{\otimes k}$, where $W=\mathbb{C}^{m n}$ is the permutation module for the symmetric group $S_{m n}$. The $G$-edge colored partition algebra $\vec{P}_{k}(n, G)$ has been introduced in [1] by Bloss, and has been realized as the centralizer algebra

Communicated by S.K. Jain.

Research supported by Govt. of India UGC-SAP and DST Research Project No. SR/FTP/MS-007/2010.

A. Joseph Kennedy $(\varangle) \cdot$ G. Muniasamy

Department of Mathematics, Ramanujan School of Mathematics and Computer Science,

Pondicherry University, Pondicherry 605 014, India

e-mail: kennedy.pondi@gmail.com 
of the wreath product $G<S_{n}$ inside $S_{|G|} \prec S_{n}$. The $G$-vertex colored partition algebra $P_{k}(n, G)$ has been introduced in [11] and has been realized as the centralizer algebra of the subgroup $G \times S_{n}$ of $G$ ? $S_{n}$. The extended vertex colored partition algebras $\widehat{P}_{k}(n, G)$, which is the centralizer algebra of the subgroup $S_{n}$ of $G \times S_{n}$, and the representations of these algebras have been studied in [12] and [13]. In the case $|G|=m$, the natural inclusion of groups $S_{n} \subseteq G \times S_{n} \subseteq G$ ? $S_{n} \subseteq S_{m}$ 々 $S_{n} \subseteq S_{m n}$ induces the natural revers inclusion of the corresponding centralizer algebras as $P_{k}(m n) \subseteq$ $P_{k}(m, n) \subseteq \vec{P}_{k}(n, G) \subseteq P_{k}(n, G) \subseteq \widehat{P}_{k}(n, G)$, which have been studied explicitly in $[6]$.

The rook (or half) partition algebras have been introduced by Martin and Rollet [9], also studied by Halverson and Ram [3] and Grood [2] with different notations. We will use the notation $P_{k+\frac{1}{2}}(x)$ for the half partition algebra. The half partition algebra $P_{k+\frac{1}{2}}(x)$ is the centralizer algebra of the symmetric group $S_{n-1}$ on $V^{\otimes k}$, where $V=\mathbb{C}^{n}$ is the natural representation of $S_{n}$. This rook version is used to construct the RSK correspondence for the partition algebra, see [4]. In this paper, we study the rook version of these colored partition algebras $P_{k}(x, G)$ and $\widehat{P}_{k}(n, G)$ and the corresponding Schur-Weyl dualities.

\section{Preliminaries}

2.1 The structure of $P_{k}(x)$ and the rook version

A $k$-partition diagram is a simple graph on two rows of $k$-vertices, one above the other. The connected components of such a graph partition the $2 k$ vertices into $l$ disjoint subsets with $1 \leq l \leq 2 k$. We say that two $k$-diagrams are equivalent if they give rise to the same partition of the $2 k$ vertices. For example, the following are equivalent 5-diagrams.

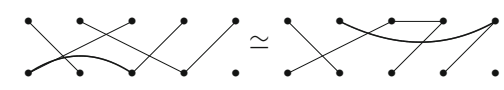

When we speak of diagrams, we are really talking about the associative equivalence classes. Number the vertices of a $k$-diagram $1,2, \ldots, k$ from left to right in the top row, and $k+1, k+2, \ldots, 2 k$ from left to right in the bottom row.

For every field $F$ and $x \in F$, we can define the partition algebra $P_{k}(x)$ on $F$-span of the $k$-partition diagrams with the following multiplication on diagrams.

The multiplication of two $k$-partition diagrams $d$ and $d^{\prime}$ is defined as follows:

- Place $d$ on the top and $d^{\prime}$ at the bottom.

- Identify (or join) the $(k+j)^{\text {th }}$ vertex of $d$ with the $j^{\text {th }}$ vertex of $d^{\prime}$. The resulting diagram now has a top row, a bottom row and a middle row of vertices.

- Let $d^{\prime \prime}$ be the partition diagram whose classes are obtained from the resulting diagram by using only the top and bottom row vertices in which they are connected by some path. Replace each "component" which is contained in the middle level by the variable $x$. (ie.) $d d^{\prime}=x^{\lambda} d^{\prime \prime}$, where $\lambda$ is the number of components in the middle level. 
For example,

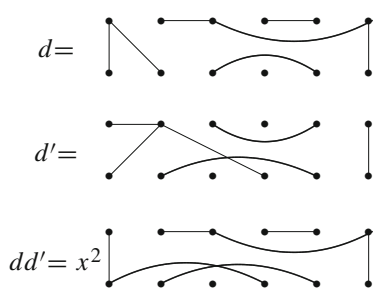

This product is associative and is independent of the graph that we choose to represent the $k$-partition diagram. The identity is given by the partition diagram having each vertex in the top row connected to the vertex below it in the bottom row. The dimension of $P_{k}(x)$ is the Bell number $B(2 k)$ and

$$
B(2 k)=\sum_{l=1}^{l=2 k} S(2 k, l),
$$

where the Sterling number $S(2 k, l)$ is the number of equivalence relations having exactly $l$ parts.

The span of the partition diagrams for which each component has exactly two vertices is the Brauer algebra $B_{k}(x)$. The span of the partition diagrams for which each component has exactly two vertices, one in each row is the group algebra $F\left[S_{k}\right]$ of the symmetric group $S_{k}$.

For $1 \leq i \leq k-1$ and $1 \leq j \leq k$, define

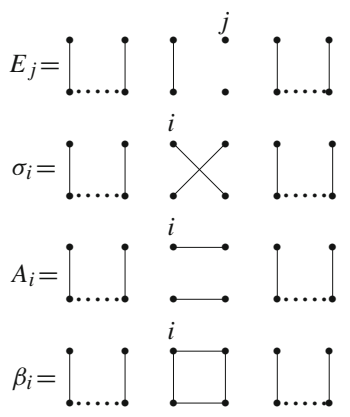

The elements $\left\{\sigma_{i}\right\}$ generate $F\left[S_{k}\right]$, the elements $\left\{\sigma_{i}, A_{i}\right\}$ generate the $B_{k}(x)$ and the elements $\left\{\sigma_{i}, \beta_{i}, E_{j}\right\}$ generate $P_{k}(x)$.

Theorem 2.1.1 [10] For each integer $k \geq 0, P_{k}(x)$ is semisimple over $\mathbb{C}(x)$, the field of complex rational polynomials in $x$. The algebra $P_{k}(\xi)$ is semisimple over $\mathbb{C}$ whenever $\xi$ is not an integer in the range [0,2k-1].

\subsection{Schur-Weyl duality}

Let $V=\mathbb{C}^{n}$ with standard basis $v_{1}, v_{2}, \ldots, v_{n}$ be the permutation module for the symmetric group $S_{n}$. Then $\pi\left(v_{i}\right)=v_{\pi(i)}$, for $\pi \in S_{n}$ and $1 \leq i \leq n$. For each 
positive integer $k$, the tensor product space $V^{\otimes k}$ is a module for the group $S_{n}$ with a standard basis given by $v_{i_{1}} \otimes v_{i_{2}} \otimes \cdots \otimes v_{i_{k}}$, where $1 \leq i_{j} \leq n$. The action of $\pi \in S_{n}$ on a basis vector is given by

$$
\pi\left(v_{i_{1}} \otimes v_{i_{2}} \otimes \cdots \otimes v_{i_{k}}\right)=v_{\pi\left(i_{1}\right)} \otimes v_{\pi\left(i_{2}\right)} \otimes \cdots \otimes v_{\pi\left(i_{k}\right)}
$$

For each $k$-partition diagram $d$ and each integer sequence $i_{1}, i_{2}, \ldots, i_{2 k}$ with $1 \leq i_{s} \leq$ $n$, define

$\psi(d)_{i_{k+1}, \ldots, i_{2 k}}^{i_{1}, i_{2}, \ldots, i_{k}}= \begin{cases}1 & \text { if } i_{r}=i_{s} \text { whenever } r \sim s \text { (i.e. } r \text { and } s \text { are in same class) in } d \\ 0 & \text { otherwise. }\end{cases}$

Define the action of a partition diagram $d \in P_{k}(n)$ on $V^{\otimes k}$ by defining it on the standard basis by

$$
d\left(v_{i_{1}} \otimes v_{i_{2}} \otimes \cdots \otimes v_{i_{k}}\right)=\sum_{i k+1, \ldots, i_{2 k}} \psi(d)_{i_{k+1}, \ldots, i_{2 k}}^{i_{1}, i_{2}, \ldots, i_{k}} v_{i_{k+1}} \otimes v_{i_{k+2}} \otimes \cdots \otimes v_{i_{2 k}}
$$

Theorem 2.2.1 (Jones [5]) $S_{n}$ and $P_{k}(n)$ generate full centralizers of each other in $\operatorname{End}\left(V^{\otimes k}\right)$. In particular, for $n \geq 2 k$, (a) $P_{k}(n) \cong \operatorname{End}_{S_{n}}\left(V^{\otimes k}\right)$. (b) $S_{n}$ generates $\operatorname{End}_{P_{k}(n)}\left(V^{\otimes k}\right)$.

The rook partition algebra $P_{k+\frac{1}{2}}(n)$ is the centralizer algebra of the subgroup $S_{n-1}$ of all permutation fixing $n$ in $S_{n}$. Hence we have $P_{k}(n) \subseteq P_{k+\frac{1}{2}}(n)$. The rook partition algebra $P_{k+\frac{1}{2}}(n)$ has been realized as a subalgebra of the partition algebra $P_{k+1}(n)$ as the span of all partition diagrams in which the last two vertices $(k+1$ th and $2(k+1)$ th) are in a same class, see for example $[2,3,9]$.

\subsection{The colored partition algebras $P_{k}(x, G)$}

Let $G$ be a group. We denote $[m]$ for the set $\{1,2, \ldots, m\}$. Let $G^{2 k}=\{f \mid f$ : $[2 k] \longrightarrow G\}$. We say that each $f \in G^{2 k}$ is a coloring of [2k] by $G$. We define a multiplication on $G^{2 k}$ by $f f^{\prime}(p)=f(p) f^{\prime}(p)$, for all $f, f^{\prime} \in G^{2 k}$ and $p \in[2 k]$. Note that under this multiplication, $G^{2 k}$ is a group, called the coloring group of [2k] by $G$.

Let $f \in G^{2 k}$. We can write $f=\left(f_{1}, f_{2}\right)$, where $f_{1}, f_{2} \in G^{k}$ are defined on $[k]$ by $f_{1}(p)=f(p), f_{2}(p)=f(k+p)$, for all $p \in[k]$. We say that $f_{1}$ and $f_{2}$ are the first and the second component of $f$ respectively.

A $(G, k)$-vertex colored partition diagram (or simply $G$-diagram) is a $k$-partition diagram, where each vertex is labelled by an element of the group $G$. We can identify each $G$-diagram as a pair $(d, f)$, where $d$ is the underlying $k$-partition diagram and $f \in G^{2 k}$ such that $f(i)$ is the label of the $i$ th vertex.

Let $(d, f)$ and $\left(d^{\prime}, f^{\prime}\right)$ be two $G$-diagrams, where $d, d^{\prime}$ are any two $k$-partition diagrams and $f=\left(f_{1}, f_{2}\right), f^{\prime}=\left(f_{1}^{\prime}, f_{2}^{\prime}\right) \in G^{2 k}$. In [11], we defined an equivalence 
relation $\sim$ on $G$-diagrams and a multiplication on $G$-diagrams, which is associative and well-defined up to equivalence of such diagrams, as follows:

- $\quad(d, f) \sim\left(d^{\prime}, f^{\prime}\right) \Leftrightarrow d \sim d^{\prime}$ and $f=\bar{g} f^{\prime}$ for some (unique) $\bar{g} \in \bar{G}$ $\Leftrightarrow d \sim d^{\prime}$ and $f \in \bar{G} f^{\prime}$.

- $\quad\left(d^{\prime}, f^{\prime}\right)(d, f)=\left\{\begin{array}{ll}x^{\lambda}\left(d^{\prime \prime}, f^{\prime \prime}\right) & \text { if } f_{2}=\left(\bar{g} f^{\prime}\right)_{1} \text { for some (unique) } \bar{g} \in \bar{G} \\ 0 & \text { otherwise, }\end{array}\right.$ where $d^{\prime} d=x^{\lambda} d^{\prime \prime}$ and $f^{\prime \prime}=\left(f_{1},\left(\bar{g} f^{\prime}\right)_{2}\right)$.

When we speak of a $G$-diagram, we are really speaking of its equivalence class. The $F$-span of all $\sim$-classes of $G$-diagrams is denoted as $P_{k}(x, G)$, called the $G$-vertex colored partition algebra, which is an associative algebra with identity. For each $\sim$ class, we can choose a $G$-diagram $(d, f)$ such that $f(1)=e$. Now we may consider the set $\{(d, f) \mid f(1)=e\}$ as a basis for the algebra $P_{k}(x, G)$. The identity in $P_{k}(x, G)$ is

$$
\sum_{\substack{f \in G^{2 k} \\ f(1)=e, f_{1}=f_{2}}}(d, f),
$$

where $d$ is the identity partition diagram. The dimension of the algebra $P_{k}(x, G)$ is $|G|^{2 k-1} B(2 k)$, if $G$ is finite.

Let $G$ be any finite group of order $m$ and let $W$ be the natural permutation module for the symmetric group $S_{m n}$ of dimension $m n$. We can identify $W$ as $\operatorname{Span}_{\mathbb{C}}\left\{v_{(i, g)} / 1 \leq\right.$ $i \leq n$ and $g \in G\}$. In [11], we defined a map $\phi: P_{k}(n, G) \longrightarrow \operatorname{End}\left(W^{\otimes k}\right)$ by defining it on a basis element $(d, f)$ such that $f(1)=e$, as follows:

$$
\begin{aligned}
\phi(d, f) & =\left(\phi(d, f)_{\left(i_{k+1}, h_{k+1}\right),\left(i_{k+2}, h_{k+2}\right), \ldots,\left(i_{2 k}, h_{2 k}\right)}^{\left(i_{1}, h_{1}\right),\left(i_{2}, h_{2}\right), \ldots,\left(i_{k}, h_{k}\right)}\right) \\
& =\left(\psi(d)_{i_{k+1}, i_{k+2}, \ldots, i_{2 k}}^{i_{1}, i_{2}, \ldots, i_{k}} \delta_{h_{1}, h_{2}, \ldots, h_{2 k}}^{h_{1}(f(1), f(2), \ldots, f(2 k))}\right) \\
& =\sum_{\substack{g \in G \\
p \sim q \text { in } d \Rightarrow i_{p}=i_{q}}} E_{\left(i_{k+1}, g f(k+1)\right),\left(i_{k+2}, g f(k+2)\right), \ldots,\left(i_{2 k}, g f(2 k)\right)}^{\left(i_{1}, g f(1)\right),\left(i_{2}, g f(2)\right), \ldots,\left(i_{k}, g f(k)\right)}
\end{aligned}
$$

where $\psi(d)_{i_{k+1}, i_{k+2}, \ldots, i_{2 k}}^{i_{1}, i_{2}, \ldots, i_{k}}$ is defined as in Eq. (2.3). We have an action of the algebra $P_{k}(n, G)$ on $W^{\otimes k}$ with respect to $\phi$, defined by

$$
\begin{aligned}
& (d, f) .\left(v_{\left(i_{1}, h_{1}\right)} \otimes v_{\left(i_{2}, h_{2}\right)} \otimes \cdots \otimes v_{\left(i_{k}, h_{k}\right)}\right)=\delta_{\left(h_{1}, h_{2}, \ldots h_{2 k}\right)}^{h_{1}(e, f(2), f(3), \ldots, f(2 k))} \\
& \quad \times \sum_{1 \leq i_{k+1}, i_{k+2}, \ldots, i_{2 k} \leq n} \psi(d)_{i_{k+1}, i_{k+2}, \ldots, i_{2 k}}^{i_{1}, i_{2}, \ldots, i_{k}} v_{\left(i_{k+1}, h_{k+1}\right)} \otimes \cdots \otimes v_{\left(i_{2 k}, h_{2 k}\right)} .
\end{aligned}
$$

Consider the restricted action (as explained in the introduction) of the subgroup $G \times S_{n}$ on $W$ as follows: $\pi_{g}(i, h)=(\pi(i), g h)$. Then $\phi$ is a algebra homomorphism onto $\operatorname{End}_{G \times S_{n}}\left(W^{\otimes k}\right)($ see, [11]).

Theorem 2.3.1 [11] $\mathbb{C}\left[G \times S_{n}\right]$ and $P_{k}(n, G)$ generate full centralizers of each other in $\operatorname{End}\left(W^{\otimes k}\right)$. In particular, for $n \geq 2 k$ 
(a) $P_{k}(n, G) \cong \operatorname{End}_{G \times S_{n}}\left(W^{\otimes k}\right)$

(b) $G \times S_{n}$ generates End $_{P_{k}(n, G)}\left(W^{\otimes k}\right)$.

The another algebra $\widehat{P}_{k}(n, G)$ is spanned by all $G$-diagrams with the following multiplication:

- $\left(d^{\prime}, f^{\prime}\right)(d, f)= \begin{cases}x^{\lambda}\left(d^{\prime \prime}, f^{\prime \prime}\right) & \text { if } f_{2}=f_{1}^{\prime} \\ 0 & \text { otherwise, }\end{cases}$

where $d^{\prime} d=x^{\lambda} d^{\prime \prime}$ and $f^{\prime \prime}=\left(f_{1},\left(\bar{g} f^{\prime}\right)_{2}\right)$.

Theorem 2.3.2 [12] $\mathbb{C}\left[S_{n}\right]$ and $\widehat{P}_{k}(n, G)$ generate full centralizers of each other in $\operatorname{End}\left(W^{\otimes k}\right)$. In particular, for $n \geq 2 k$

(a) $\widehat{P}_{k}(n, G) \cong \operatorname{End}_{S_{n}}\left(W^{\otimes k}\right)$

(b) $S_{n}$ generates $\operatorname{End}_{\widehat{P}_{k}(n, G)}\left(W^{\otimes k}\right)$.

\section{The rook version of colored partition algebras}

In this section, we introduce the rook version of the colored partition algebras and study its structure.

\subsection{Two bases for $E n d_{G \times S_{n-1}}\left(W^{\otimes k}\right)$ and $E n d_{S_{n-1}}\left(W^{\otimes k}\right)$}

In this section, we give two bases for $E n d_{G \times S_{n-1}}\left(W^{\otimes k}\right)$, where $W=\mathbb{C}^{|G| n}$ and the action of $G \times S_{n-1}$ on $W^{\otimes k}$ is defined as follows:

Let $G$ be any finite group and let $W$ be a vector space of dimension $|G| n$. We can identify $W$ as $\operatorname{Span}_{\mathbb{C}}\left\{v_{(i, g)} / 1 \leq i \leq n\right.$ and $\left.g \in G\right\}$. Note that when $G$ is the group with one element, $W$ specializes to $V$, the permutation representation of $S_{n}$. The action of $G \times S_{n-1}$ on $W$ is defined as

$$
\pi_{g}\left(v_{(i, h)}\right)=v_{(\pi(i), g h)}, \forall \pi \in S_{n-1} \text { and } g \in G
$$

(note that $S_{n-1}$ is the subgroup of all permutation fixing $n$ in $S_{n}$ ).

Diagonally extend the action of $G \times S_{n-1}$ on $W$ to an action of $G \times S_{n-1}$ on $W^{\otimes k}$ :

$$
\pi_{g}\left(v_{\left(i_{1}, g_{1}\right)} \otimes \cdots \otimes v_{\left(i_{k}, g_{k}\right)}\right)=v_{\left(\pi\left(i_{1}\right), g g_{1}\right)} \otimes \cdots \otimes v_{\left(\pi\left(i_{k}\right), g g_{k}\right)}
$$

where $\pi \in S_{n-1}$ and $g \in G$. We will write above as $\pi_{g}\left(v_{I}\right)=v_{\pi_{g}(I)}$.

Let $A \in \operatorname{End}\left(W^{\otimes k}\right)$. Define $A\left(v_{I}\right)=\sum_{J} A_{J}^{I}\left(v_{J}\right)$, where $A_{J}^{I} \in \mathbb{C}$ is the $(I, J)^{\text {th }}$ entry of $A ; I, J \in \mathbb{S}^{k}$, where $\mathbb{S}=[n] \times G$ and $v_{J}$ is the basis element of $W^{\otimes k}$. We have

$$
\operatorname{End}_{G \times S_{n}}\left(W^{\otimes k}\right) \subseteq \operatorname{End}_{G \times S_{n-1}}\left(W^{\otimes k}\right) \subseteq \operatorname{End}_{S_{n-1}}\left(W^{\otimes k}\right)
$$

The following is our analogue of Jones result. 
Lemma 3.1.1 (a) $A \in \operatorname{End}_{G \times S_{n-1}}\left(W^{\otimes k)} \Leftrightarrow A_{J}^{I}=A_{\pi(J)}^{\pi(I)}, \forall \pi_{g} \in G \times S_{n-1}\right.$. (b) $A \in \operatorname{End}_{S_{n-1}}\left(W^{\otimes k)} \Leftrightarrow A_{J}^{I}=A_{\pi(J)}^{\pi(I)}, \forall \pi \in S_{n-1}\right.$.

Proof (a) We have $A \in \operatorname{End}_{G \times S_{n-1}}\left(W^{\otimes k}\right) \Leftrightarrow \pi_{g} A=A \pi_{g}, \forall \pi_{g} \in G \times S_{n-1}$.

$\Leftrightarrow \pi_{g} A\left(v_{I}\right)=A \pi_{g}\left(v_{I}\right) \forall v_{I}$.

$\Leftrightarrow \pi_{g} \sum_{J} A_{J}^{I}\left(v_{J}\right)=A\left(v_{\pi_{g}(I)}\right)$

$\Leftrightarrow \sum_{J} A_{J}^{I} \pi_{g}\left(v_{J}\right)=\sum_{J} A_{J}^{\pi_{g}(I)}\left(v_{J}\right)$

$\Leftrightarrow \sum_{J} A_{J}^{I}\left(v_{\pi_{g}(J)}\right)=\sum_{J} A_{\pi_{g}(J)}^{\pi_{g}(I)}\left(v_{\pi_{g}(J)}\right)$, since the action of $G \times S_{n-1}$ is the permutation representation. The result (a) follows from linear independence and equating the scalars. The proof of (b) is similar to the proof of (a).

\section{Lemma 3.1.2}

(a) $\operatorname{dim} E n d_{G \times S_{n-1}}\left(W^{\otimes k}\right)=|G|^{2 k-1} \sum_{l=1}^{l=n} S(2 k+1, l)$.

When $n-1 \geq 2 k$,

$$
\begin{aligned}
& \operatorname{dim} \text { End }_{G \times S_{n-1}}\left(W^{\otimes k}\right)=|G|^{2 k-1} B(2 k+1) . \\
& \text { (b) } \operatorname{dim} \operatorname{End}_{S_{n-1}}\left(W^{\otimes k}\right)=|G|^{2 k} \sum_{l=1}^{l=n} S(2 k+1, l) .
\end{aligned}
$$

When $n-1 \geq 2 k$,

$$
\operatorname{dim} \operatorname{End}_{S_{n-1}}\left(W^{\otimes k}\right)=|G|^{2 k} B(2 k+1) .
$$

Proof (a) The lemma above tells us that $A$ commutes with the $G \times S_{n-1}$-action on $W^{\otimes k}$ if and only if the matrix entries of $A$ are equal on the $G \times S_{n-1}$-orbits. Thus $\operatorname{dim} \operatorname{End}_{G \times S_{n-1}}\left(W^{\otimes k}\right)$ is the number of $G \times S_{n-1}$-orbits on $\mathbb{S}^{2 k}=\left\{\left(\left(i_{1}, g_{1}\right),\left(i_{2}, g_{2}\right)\right.\right.$ $\left.\ldots,\left(i_{2 k}, g_{2 k}\right)\right) \mid 1 \leq i_{r} \leq n$ and $\left.g_{r} \in G\right\}$. Fix a tuple of indices $(I, J)=\left(\left(i_{1}, g_{1}\right)\right.$, $\left.\left(i_{2}, g_{2}\right) \ldots,\left(i_{2 k}, g_{2 k}\right)\right) \in \mathbb{S}^{2 k}$. This tuple determines a partition $d(I, J)=d\left(i_{1}, i_{2}, \ldots\right.$, $i_{2 k}$ ) of [2k] (into at most $n$ subsets) according to those that have an equal value. Let $[(I, J)]$ be the orbit of $(I, J) \in \mathbb{S}^{2 k}$.

Then $\left(I^{\prime}, J^{\prime}\right) \in[(I, J)] \Leftrightarrow\left(I^{\prime}, J^{\prime}\right)=\pi(I, J)$ for some $\pi_{g} \in G \times S_{n-1}$

$\Leftrightarrow\left(j_{r}, h_{r}\right)=\pi_{g}\left(i_{r}, g_{r}\right)$ for every $r$ such that $1 \leq r \leq 2 k$, where $\left(j_{r}, h_{r}\right)$ and $\left(i_{r}, g_{r}\right)$ are the $r^{\text {th }}$ component of $\left(I^{\prime}, J^{\prime}\right)$ and $(I, J)$ respectively

$$
\begin{aligned}
\Leftrightarrow & \left(j_{r}, h_{r}\right)=\left(\pi\left(i_{r}\right), g g_{r}\right) \\
\Leftrightarrow & j_{r}=\pi\left(i_{r}\right) \text { and } h_{r}=g g_{r} \\
\Leftrightarrow & {\left[j_{p}=j_{q} \text { iff } i_{p}=i_{q}(1 \leq p, q \leq 2 k)\right], \quad\left[j_{p}=n \text { iff } i_{p}=n(1 \leq p \leq 2 k)\right] } \\
& \text { and } h_{r}=g g_{r}(1 \leq r \leq 2 k)
\end{aligned}
$$

$\Leftrightarrow d\left(j_{1}, j_{2}, \ldots, j_{2 k}\right)=d\left(i_{1}, i_{2}, \ldots, i_{2 k}\right),\left[j_{p}=n\right.$ iff $\left.i_{p}=n(1 \leq p \leq 2 k)\right]$ and $h_{r}=g g_{r} \forall r,(1 \leq r \leq 2 k)$.

Thus, for every $G \times S_{n-1}$-orbit $[(I, J)]$ determines a partition $d=d\left(i_{1}, i_{2}, \ldots, i_{2 k}\right)$ (into at most $n$ subsets) and a class $N=\left\{p \in[2 k] \mid i_{p}=n\right\}$ of $d$ and a $2 k$-tuple $f=\left(e, g_{2}, \ldots, g_{2 k}\right)$ (i.e a pair $\left(d_{N}, f\right)$ such that $f(1)=e$ ) and vice versa. Hence the result (a) is proved. 
Similarly, for every $S_{n-1}$-orbit $[(I, J)]$ determines a partition $d=d\left(i_{1}, i_{2}, \ldots, i_{2 k}\right)$ (into at most $n$ subsets) and a class $N=\left\{p \in[2 k] \mid i_{p}=n\right\}$ of $d$ and a $2 k$-tuple $f=\left(g_{1}, g_{2}, \ldots, g_{2 k}\right)$ (i.e a pair $\left(d_{N}, f\right)$ ) and vice versa. Hence the result (b) is proved.

We define for each $G \times S_{n-1}$-orbit $\left(d_{N}, f\right)=[(I, J)]$, a matrix $T_{J}^{I} \in \operatorname{End}\left(W^{\otimes k}\right)$ by $T_{J}^{I}=\sum_{\left(I^{\prime}, J^{\prime}\right) \in[(I, J)]} E_{J^{\prime}}^{I^{\prime}}$, where $E_{J^{\prime}}^{I^{\prime}}$ is the matrix unit, which has non-zero entry 1 in the $\left(I^{\prime}, J^{\prime}\right)^{t h}$ position. In fact, $T_{J}^{I} \in \operatorname{End}\left(W^{\otimes k}\right)$, since such a matrix satisfies the condition in Lemma 3.1.1 : the matrix entries are equal on the $G \times S_{n-1}$-orbits. Using Eq. (3.2), we have

$$
T_{\left(i_{k+1}, g_{k+1}\right),\left(i_{k+2}, g_{k+2}\right), \ldots,\left(i_{2 k}, g_{2 k}\right)}^{\left(i_{1}, g_{1}\right),\left(i_{2}, g_{2}\right), \ldots,\left(i_{k}, g_{k}\right)}=\sum E_{\left(j_{k+1}, g g_{k+1}\right),\left(j_{k+2}, g g_{k+2}\right), \ldots,\left(j_{2 k}, g g_{2 k}\right)}^{\left(j_{1}, g g_{1}\right),\left(j_{2}, g g_{2}\right), \ldots,\left(j_{k}, g g_{k}\right)}
$$

where the sum is over $g \in G$ and $i_{p}=i_{q} \Leftrightarrow j_{p}=j_{q},(1 \leq p, q \leq 2 k)$ [i.e $p \sim q$ in $\left.P\left(i_{1}, i_{2}, \ldots, i_{2 k}\right) \Leftrightarrow j_{p}=j_{q}\right]$ and $i_{p}=n \Leftrightarrow j_{p}=n$.

Since each matrix $T_{J}^{I}$ is the sum of disjoint sets of matrix units, the set $\left\{T_{J}^{I} /[(I, J)]\right.$ is a $G \times S_{n-1}-$ orbit\} is a linearly independent set. For $A \in E n d_{G \times S_{n-1}}\left(W^{\otimes k}\right)$, we use the Lemma 3.1.1 to obtain: $A=\sum_{[(I, J)]} A_{J}^{I} T_{J}^{I}$. Thus the matrix $T_{J}^{I}$ span $E n d_{G \times S_{n-1}}\left(W^{\otimes k}\right)$, so is a basis for $E n d_{G \times S_{n-1}}\left(W^{\otimes k}\right)$.

Definition 3.1.3 Let $d$ and $d^{\prime}$ be partitions of [2k] into subsets. We say that $d^{\prime}$ is coarser than $d$ if any subset in $d$ is contained in some subset in $d^{\prime}$. In this case we write $d^{\prime} \leq d$.

We now define another basis of $E n d_{G \times S_{n-1}}\left(W^{\otimes k}\right)$ as follows : Define $L_{J}^{I}=\sum T_{J^{\prime}}^{I^{\prime}}$, the sum is over $\left[\left(I^{\prime}, J^{\prime}\right)\right]$ such that $d\left[\left(I^{\prime}, J^{\prime}\right)\right] \leq d[(I, J)]$. By Möbius inversion the $T_{J}^{I}$ can be expressed in terms of the $L_{J}^{I}$ 's so they also span $E n d_{G \times S_{n-1}}\left(W^{\otimes k}\right)$. Using Eq. (3.3), we get

$$
L_{\left(i_{k+1}, g_{k+1}\right),\left(i_{k+2}, g_{k+2}\right), \ldots,\left(i_{2 k}, g_{2 k}\right)}^{\left(i_{1}, g_{1}\right),\left(i_{2}, g_{2}\right), \ldots,\left(i_{k}, g_{k}\right)}=\sum E_{\left(j_{k+1}, g g_{k+1}\right),\left(j_{k+2}, g g_{k+2}\right), \ldots,\left(j_{2 k}, g g_{2 k}\right)}^{\left(j_{1}, g g_{1}\right),\left(j_{2}, g g_{2}\right), \ldots,\left(j_{k}, g g_{k}\right)}
$$

where the sum is over $g \in G$ and $i_{p}=i_{q} \Rightarrow j_{p}=j_{q}(1 \leq p, q \leq 2 k)$ and $i_{p}=n \Rightarrow j_{p}=n$.

Similarly, we use the proof of Lemma 3.1.2(b) to define for each $S_{n-1}$-orbit $[(I, J)]$ a matrix in $\operatorname{End}_{S_{n-1}}\left(W^{\otimes k}\right)$, as follows:

$$
\widetilde{L}_{\left(i_{k+1}, g_{k+1}\right),\left(i_{k+2}, g_{k+2}\right), \ldots,\left(i_{2 k}, g_{2 k}\right)}^{\left(i_{1}, g_{1}\right),\left(i_{2}, g_{2}\right), \ldots,\left(i_{k}, g_{k}\right)}=\sum E_{\left(j_{k+1}, g_{k+1}\right),\left(j_{k+2}, g_{k+2}\right), \ldots,\left(j_{2 k}, g_{2 k}\right)}^{\left(j_{1}, g_{1}\right),\left(j_{2}, g_{2}\right), \ldots,\left(j_{k}, g_{k}\right)}
$$

where the sum is over $i_{p}=i_{q} \Rightarrow j_{p}=j_{q}(1 \leq p, q \leq 2 k)$ and $i_{p}=n \Rightarrow j_{p}=n$. Note that

$$
L_{\left(i_{k+1}, g_{k+1}\right),\left(i_{k+2}, g_{k+2}\right), \ldots,\left(i_{2 k}, g_{2 k}\right)}^{\left(i_{1} g_{1}\right)\left(i_{2}, g_{2}\right), \ldots,\left(i_{k}, g_{k}\right)}=\sum_{g \in G} \widetilde{L}_{\left(j_{k+1}, g g_{k+1}\right),\left(j_{k+2}, g g_{k+2}\right), \ldots,\left(j_{2 k}, g g_{2 k}\right)}^{\left(j_{1}, g g_{1}\right),\left(j_{2}, g g_{2}\right), \ldots,\left(j_{k}, g g_{k}\right)} .
$$


3.2 The structure of $P_{k+\frac{1}{2}}(x, G)$ and $\widehat{P}_{k+\frac{1}{2}}(x, G)$

A $\left(G, k+\frac{1}{2}\right)$-partition diagram is a $(G, k)$-partition diagram whose underlying partition $d$ with a special class $N$ of $d$ ( $N$ may be empty) and the vertices are colored by $G$. Now each $\left(G, k+\frac{1}{2}\right)$-diagram can be identified as a pair $\left(d_{N}, f\right)$, where $d$ is the partition of the set [2k] induced by the $(G, k)$-diagram, $N$ is the unique special class of $d$ ( $N$ may be empty) and $f \in G^{2 k}$ is the coloring induced by the coloring sequence of the $(G, k)$-diagram.

For each $d_{N}$, we can get a partition with $2 k+1$ vertices by adding a $2 k+1$ th vertex in the special class $N$ and vice versa. Hence the number of $\left(G, k+\frac{1}{2}\right)$-diagrams is $|G|^{2 k} B(2 k+1)$. Thus each $\left(G, k+\frac{1}{2}\right)$-diagram can be identified as a $(G, k)$-partition diagram with one more vertex in the right side of the diagram, which is connected with the special class $N$.

We define two multiplications on $\left(G, k+\frac{1}{2}\right)$-diagrams, where two $G$-diagrams $\left(d_{N}, f\right)$ and $\left(d_{N^{\prime}}^{\prime}, f^{\prime}\right)$, where $d, d^{\prime}$ are any two partitions of the set [2k] and $N, N^{\prime}$ are the special classes of $d, d^{\prime}$ connected with the $2 k+1$ th vertex respectively and $f=\left(f_{1}, f_{2}\right), f^{\prime}=\left(f_{1}^{\prime}, f_{2}^{\prime}\right) \in G^{2 k}$, as follows:

$$
\left(d_{N}, f\right)\left(d_{N^{\prime}}^{\prime}, f^{\prime}\right)= \begin{cases}x^{\lambda}\left(d_{N} d_{N^{\prime}}^{\prime},\left(f_{1}^{\prime}, g f_{2}\right)\right) & \text { if } f_{2}^{\prime}=g f_{1} \text { for some } g \in G \\ 0 & \text { otherwise, }\end{cases}
$$

where $\lambda$ is the number of middle components of $d_{N} d_{N^{\prime}}^{\prime}$ as in the partition algebra case.

(2)

$$
\left(d_{N}, f\right) *\left(d_{N^{\prime}}^{\prime}, f^{\prime}\right)= \begin{cases}x^{\lambda}\left(d_{N} d_{N^{\prime}}^{\prime},\left(f_{1}^{\prime}, f_{2}\right)\right. & \text { if } f_{2}^{\prime}=f_{1} \\ 0 & \text { otherwise }\end{cases}
$$

where $\lambda$ is the number of middle components of $d_{N} d_{N^{\prime}}^{\prime}$

The multiplication (1) of two $G$-diagrams $\left(d_{N}, f\right)$ and $\left(d_{N^{\prime}}^{\prime}, f^{\prime}\right)$ defined above can be equivalently stated in other words as follows:

- Multiply the underlying partition diagrams $d_{N}$ and $d_{N^{\prime}}^{\prime}$. This will give the underlying partition diagram of the $G$-diagram $\left(d_{N}, f\right)\left(d_{N^{\prime}}^{\prime}, f^{\prime}\right)$.

- If the bottom label sequence of $\left(d_{N}, f\right)$ is equal to the top label sequence of $\left(d_{N^{\prime}}^{\prime}, g f^{\prime}\right)$ for some $g \in G$ then the top label sequence and the bottom label sequence of $\left(d_{N}, f\right)\left(d_{N^{\prime}}^{\prime}, f^{\prime}\right)$ are the top label sequence of $\left(d_{N}, f\right)$ and the bottom label sequence of $\left(d_{N}^{\prime}, g f^{\prime}\right)$ respectively.

- Otherwise $\left(d_{N}, f\right)\left(d_{N^{\prime}}^{\prime}, f^{\prime}\right)=0$.

- For each connected component entirely in the middle row, a factor of $x$ (indeterminant) appears in the product.

For example for the multiplication (1), let $g_{r}, h_{s} \in G(1 \leq r, s \leq 12)$. 


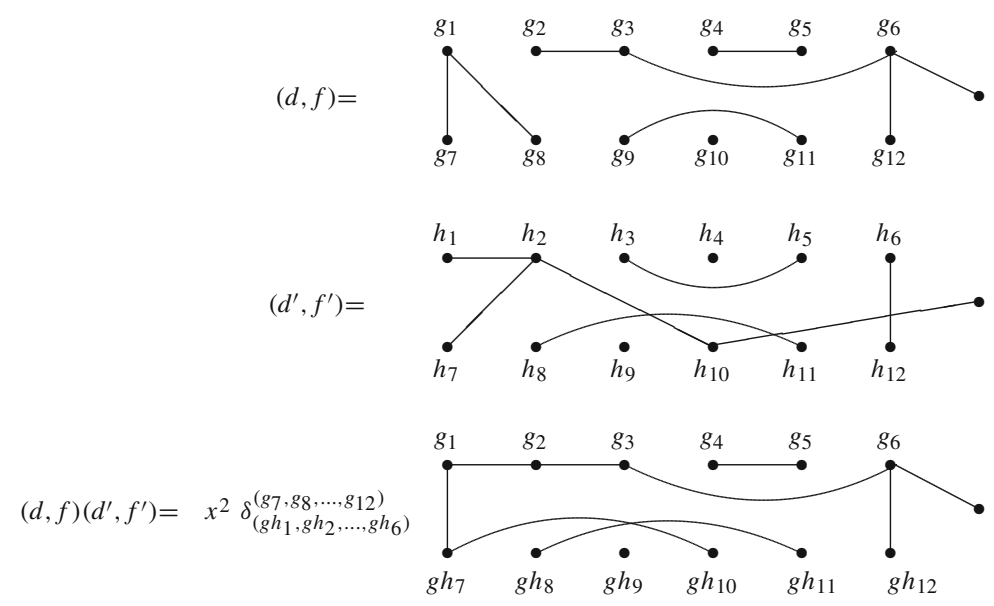

Note that $\delta$ is the Kronecker delta, that is

$$
\delta_{\left(g h_{1}, g h_{2}, \ldots, g h_{6}\right)}^{\left(g_{7}, g_{8}, \ldots, g_{12}\right)}= \begin{cases}1 & \text { if }\left(g_{7}, g_{8}, \ldots, g_{12}\right)=\left(g h_{1}, g h_{2}, \ldots, g h_{6}\right) \\ 0 & \text { if }\left(g_{7}, g_{8}, \ldots, g_{12}\right) \neq\left(g h_{1}, g h_{2}, \ldots, g h_{6}\right) .\end{cases}
$$

The multiplication (2) can be explained using diagrams in the similar way.

Moreover the multiplication (2) is well defined under the following equivalence relation:

$$
\left(d_{N}, f\right) \sim\left(d_{N^{\prime}}^{\prime}, f^{\prime}\right) \text { iff } d_{N} \sim d_{N^{\prime}}^{\prime} \text { and } f=g f^{\prime} \text { for some } g \in G \text {. }
$$

The multiplications (1) and (2) are associative on $\left(G, k+\frac{1}{2}\right)$-diagrams. The $\mathbb{C}(x)$-span of all $\left(G, k+\frac{1}{2}\right)$-diagrams under the above multiplication (1) with the above equivalence relation and the multiplication (2) are denoted as $P_{k+\frac{1}{2}}(x, G)$ and $\widehat{P}_{k+\frac{1}{2}}(x, G)$ respectively, which are associative algebras with identity. The identity in $P_{k+\frac{1}{2}}(x, G)$ is $\sum_{\substack{f \in G^{2 k} \\ f_{1}=f_{2}, f_{1}(1)=f_{2}(1)=e}}\left(d_{N}, f\right)$ and in $\widehat{P}_{k+\frac{1}{2}}(x, G)$ is $\sum_{\substack{f \in G^{2 k} \\ f_{1}=f_{2}}}\left(d_{N}, f\right)$, where $d$ is the identity partition diagram and $N$ is empty.

The dimension of $P_{k+\frac{1}{2}}(x, G)$ is the number of equivalence classes defined in (3.9) of $\left(G, k+\frac{1}{2}\right)$-diagrams, so that if $G$ is finite, $\operatorname{dim} P_{k+\frac{1}{2}}(x, G)=|G|^{2 k-1} B(2 k+1)$, where $B(2 k+1)$ is the Bell number of $2 k+1$, the number of equivalence relations of $2 k+1$ vertices. Note that $P_{k+\frac{1}{2}}(x, H)$ is a subalgebra of $P_{k+\frac{1}{2}}(x, G)$ if $H$ is a subgroup of $G$. In particular, if $H=\{e\}$ then $P_{k+\frac{1}{2}}(x, H) \simeq P_{k+\frac{1}{2}}(x)$, the rook version of the partition algebra. If $G$ is an infinite group, $P_{k+\frac{1}{2}}(x, G)$ is an infinite dimensional associative algebra. When $x=\xi \in \mathbb{C}$, we obtain the $\mathbb{C}$-algebra $P_{k+\frac{1}{2}}(\xi, G)$.

Similarly, the dimension of $\widehat{P}_{k+\frac{1}{2}}(x, G)$ is the number of $\left(G, k+\frac{1}{2}\right)$-diagrams, so that if $G$ is finite, $\operatorname{dim} \widehat{P}_{k+\frac{1}{2}}(x, G)=|G|^{2 k} B(2 k+1)$. Define elements 


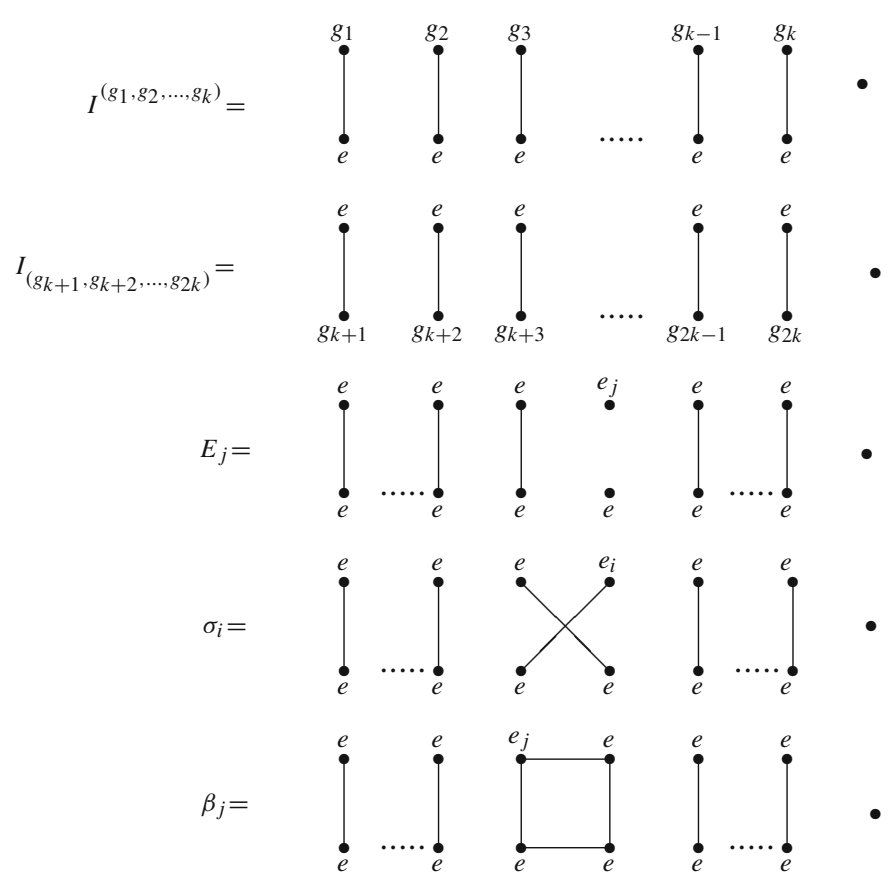

where $g_{l} \in G,(1 \leq l \leq 2 k),(2 \leq i \leq k)$ and $(1 \leq j \leq k)$. Note that the last class in $\beta_{k}$ is the triangle containing the last three vertices. We see that $\widehat{P}_{k+\frac{1}{2}}(x, G)$ is generated by the above elements and $P_{k+\frac{1}{2}}(x, G)$ is generated by the above elements except $I_{\left(g_{k+1}, g_{k+2}, \ldots, g_{2 k}\right)}$.

Theorem 3.2.1 $P_{k+\frac{1}{2}}(x, G)$ is a subalgebra of $\widehat{P}_{k+\frac{1}{2}}(x, G)$.

Proof In $\widehat{P}_{k+\frac{1}{2}}(x, G)$, for each $\left(G, k+\frac{1}{2}\right)$-diagram $\left(d_{N}, f\right)$ such that $f(1)=e$, we define the sum

$$
\left.\widehat{\left(d_{N}, f\right.}\right)=\sum_{\bar{g} \in \bar{G}}\left(d_{N}, \bar{g} f\right)
$$

In other words this sum is over all distinct $\left(G, k+\frac{1}{2}\right)$-diagrams in $\widehat{P}_{k+\frac{1}{2}}(x, G)$, which are related to the $\left(G, k+\frac{1}{2}\right)$-diagram $\left(d_{N}, f\right)$ with respect to the above equivalence relation $\sim$ on $\left(G, k+\frac{1}{2}\right)$-diagrams. So, we say that this sum is the class sum of $\left(d_{N}, f\right)$ under $\sim$ in $\widehat{P}_{k+\frac{1}{2}}(x, G)$. Since any two class sums are the disjoint sums of $\left(G, k+\frac{1}{2}\right)$-diagrams in $\widehat{P}_{k+\frac{1}{2}}(x, G)$ the set of all class sums is a linearly independent set in $\widehat{P}_{k+\frac{1}{2}}(x, G)$. We are going to prove that $\left(d_{N}, f\right) \longrightarrow\left(\widehat{d_{N}, f}\right)$ is an algebra isomorphism from $P_{k+\frac{1}{2}}(x, G)$ in to $\widehat{P}_{k+\frac{1}{2}}(x, G)$.

Now we have $\left(d_{N}, f\right)\left(d_{N^{\prime}}^{\prime}, f^{\prime}\right)=0$ in $P_{k+\frac{1}{2}}(x, G)$ 


$$
\begin{aligned}
& \Leftrightarrow f_{2}^{\prime} \neq \overline{g^{\prime}} f_{1}, \forall \overline{g^{\prime}} \in \bar{G} \\
& \Leftrightarrow\left(d_{N}, \bar{g} f\right) *\left(d_{N^{\prime}}^{\prime}, \overline{g^{\prime}} f^{\prime}\right)=0 \text { in } \widehat{P}_{k+\frac{1}{2}}(x, G), \forall \bar{g}, \overline{g^{\prime}} \in \bar{G} \\
& \Leftrightarrow\left(\sum_{\bar{g} \in \bar{G}}(d, \bar{g} f)\right) *\left(\sum_{\overline{g^{\prime}} \in \bar{G}}\left(d_{N^{\prime}}^{\prime}, \overline{g^{\prime}} f^{\prime}\right)\right)=0 \text { in } \widehat{P}_{k+\frac{1}{2}}(x, G) \\
& \Leftrightarrow\left(\widehat{d_{N}, f}\right) *\left(\widehat{d_{N^{\prime}}^{\prime}, f^{\prime}}\right)=0 \text { in } \widehat{P}_{k+\frac{1}{2}}(x, G) .
\end{aligned}
$$

Hence $\left(d_{N}, f\right)\left(d_{N^{\prime}}^{\prime}, f^{\prime}\right)=0$ in $P_{k+\frac{1}{2}}(x, G) \Leftrightarrow\left(\widehat{d_{N}, f}\right) *\left(\widehat{d_{N^{\prime}}^{\prime}, f^{\prime}}\right)=0$ in $\widehat{P}_{k+\frac{1}{2}}(x, G)$.

Suppose $\left(d_{N}, f\right)\left(d_{N^{\prime}}^{\prime}, f^{\prime}\right) \neq 0$. Let

$$
\left(d_{N}, f\right)\left(d_{N^{\prime}}^{\prime}, f^{\prime}\right)=x^{\lambda}\left(d_{N} d_{N^{\prime}}^{\prime}, f^{\prime \prime}\right),
$$

where $\lambda$ is the number of middle components in $d_{N} d_{N^{\prime}}^{\prime}$. Then

$$
\begin{aligned}
\left(\widehat{d_{N}, f}\right) *\left(\widehat{d_{N^{\prime}}^{\prime}, f^{\prime}}\right) & =\left(\sum_{\bar{g} \in \bar{G}}\left(d_{N}, \bar{g} f\right)\right) *\left(\sum_{\overline{g^{\prime}} \in \bar{G}}\left(d_{N^{\prime}}^{\prime}, \overline{g^{\prime}} f^{\prime}\right)\right) \\
& =\sum_{\bar{g}, \overline{g^{\prime}} \in \bar{G}}\left(d_{N}, \bar{g} f\right) *\left(d_{N^{\prime}}^{\prime}, \overline{g^{\prime}} f^{\prime}\right) .
\end{aligned}
$$

Note that some product $\left(d_{N}, \bar{g} f\right) *\left(d_{N^{\prime}}^{\prime}, \overline{g^{\prime}} f^{\prime}\right)$ may be 0 in the above sum (3.11). If a product $\left(d_{N}, \bar{g} f\right) *\left(d_{N^{\prime}}^{\prime}, \overline{g^{\prime}} f^{\prime}\right)$ is non zero then the underlying $\left(G, k+\frac{1}{2}\right)$-diagram must be $\sim$-equivalent to $\left(d_{N} d_{N^{\prime}}^{\prime}, f^{\prime \prime}\right)$ for some $f^{\prime \prime} \in G^{2 k}$ (using (3.10)). Also any $\left(G, k+\frac{1}{2}\right)$ - diagram which are $\sim$-equivalent to $\left(d_{N} d_{N^{\prime}}^{\prime}, f^{\prime \prime}\right)$ is of the form $\left(d_{N}, \bar{g} f\right) *$ $\left(d_{N^{\prime}}^{\prime}, \overline{g^{\prime}} f^{\prime}\right)$ for some $\bar{g}, \overline{g^{\prime}} \in \bar{G}$. Observe that if $\left(d_{N}, \bar{g} f\right) *\left(d_{N^{\prime}}^{\prime}, \overline{g^{\prime}} f^{\prime}\right) \neq 0$ then $\bar{g} f_{2}=\overline{g^{\prime}} f_{1}^{\prime}$. And hence $\left(d_{N}, \bar{g} f\right) *\left(d_{N^{\prime}}^{\prime}, \overline{g^{\prime}} f^{\prime}\right)=x^{\lambda}\left(d_{N} d_{N^{\prime}}^{\prime}, \bar{h} f^{\prime \prime}\right)$ for some $\bar{h} \in \bar{G}$. Hence (3.11) can be written as

$$
\sum_{\bar{h} \in \bar{G}} x^{\lambda}\left(d_{N} d_{N^{\prime}}^{\prime}, \bar{h} f^{\prime \prime}\right)=\left(d_{N}, \widehat{f)\left(d_{N^{\prime}}^{\prime}\right.}, f^{\prime}\right) .
$$

Thus $\left(\widehat{d_{N}, f}\right) *\left(\widehat{d_{N^{\prime}}^{\prime}, f^{\prime}}\right)=\left(d_{N}, \widehat{f)\left(d_{N^{\prime}}^{\prime}\right.}, f^{\prime}\right)$.

Hence $\operatorname{Span}_{\mathbb{C}(x)}\left\{\left(\widehat{d_{N}, f}\right) \mid\left(d_{N}, f\right)\right.$ is a $\left(G, k+\frac{1}{2}\right)$-diagram such that $\left.f(1)=e\right\}$ is a subalgebra and the map $\left(d_{N}, f\right) \longrightarrow\left(\widehat{d_{N}, f}\right)$ is an algebra isomorphism from $P_{k+\frac{1}{2}}(x, G)$ in to $\widehat{P}_{k+\frac{1}{2}}(x, G)$.

\subsection{Schur-Weyl duality}

We have the diagonal action of $G \times S_{n-1}$ on $W^{\otimes k}$, where $W$ is the permutation representation of $S_{n}$. Also, we have an action of $\widehat{P}_{k+\frac{1}{2}}(n, G)$ on $W^{\otimes k}$, defined as follows: Define a map $\widehat{\phi}: \widehat{P}_{k+\frac{1}{2}}(n, G) \longrightarrow \operatorname{End}\left(W^{\otimes k}\right)$ by defining it on a $\left(G, k+\frac{1}{2}\right)$ - diagram $\left(d_{N}, f\right)$, where $f=\left(g_{1}, g_{2}, \ldots, g_{k}, g_{k+1}, g_{k+2}, \ldots, g_{2 k}\right)$ is the label sequence of $d_{N}$ as follows: 


$$
\widehat{\phi}\left(d_{N}, f\right)=\sum_{\substack{p \sim q \\ p \in N \Rightarrow i_{p}=n \\ p \in i_{p}=i_{q}}} E_{\left(i_{k+1}, g_{k+1}\right),\left(i_{k+2}, g_{k+2}\right), \ldots,\left(i_{2 k}, g_{2 k}\right)}^{\left(i_{1}, g_{1}\right),\left(i_{2}, g_{2}\right), \ldots,\left(i_{k}, g_{k}\right)} .
$$

Then we have an action of $\widehat{P}_{k+\frac{1}{2}}(n, G)$ on $W^{\otimes k}$ defined by

$$
d\left(v_{I}\right)=\widehat{\phi}(d)\left(v_{I}\right), \forall I \in \mathbb{S}^{k}
$$

When $G$ is a group with one element, this action restricts to the action of the rook partition algebra on tensors.

The multiplication of the matrices $\widehat{L}_{J}^{I}$ in the basis of $E n d_{S_{n-1}}\left(W^{\otimes k}\right)$ has a nice form as follows:

\section{Lemma 3.3.1}

(a) $\quad\left(L_{\left.\left(i_{k+1}, g_{k+1}\right),\left(i_{k+2}, g_{k+2}\right), \ldots, i_{2 k}, g_{2 k}\right)}^{\left(i_{1}, g_{1}\right),\left(i_{2}, g_{2}\right), \ldots,\left(i_{k}, g_{k}\right)}\right)\left(L_{\left(j_{k+1}, h_{k+1}\right),\left(j_{k+2}, h_{k+2}\right), \ldots,\left(j_{2 k}, h_{2 k}\right)}^{\left(j_{1}, h_{1}\right),\left(j_{2}, h_{2}\right), \ldots,\left(j_{k}, h_{k}\right)}\right)=0$ $\Leftrightarrow g\left(g_{1}, g_{2}, \ldots, g_{k}\right) \neq\left(h_{k+1}, h_{k+2}, \ldots, h_{2 k}\right)$ for someg $\in G$.

(b) $\left(\widetilde{L}_{\left(i_{k+1}, g_{k+1}\right),\left(i_{k+2}, g_{k+2}\right), \ldots,\left(i_{2 k}, g_{2 k}\right)}^{\left(i_{1}, g_{1}\right),\left(i_{2}, g_{2}\right), \ldots,\left(i_{k}, g_{k}\right)}\right)\left(\widetilde{L}_{\left(j_{k+1}, h_{k+1}\right),\left(j_{k+2}, h_{k+2}\right), \ldots,\left(j_{2 k}, h_{2 k}\right)}^{\left(j_{1}, h_{1}\right),\left(j_{2}, h_{2}\right), \ldots,\left(j_{k}, h_{k}\right)}\right)=0$ $\Leftrightarrow\left(g_{1}, g_{2}, \ldots, g_{k}\right) \neq\left(h_{k+1}, h_{k+2}, \ldots, h_{2 k}\right)$.

\section{Proof a)}

$$
\begin{aligned}
& \left(L_{\left(i_{k+1}, g_{k+1}\right),\left(i_{k+2}, g_{k+2}\right), \ldots,\left(i_{2 k}, g_{2 k}\right)}^{\left(i_{1}, g_{1}\right),\left(i_{2}, g_{2}\right), \ldots,\left(i_{k}, g_{k}\right)}\right)\left(L_{\left(j_{k+1}, h_{k+1}\right),\left(j_{k+2}, h_{k+2}\right), \ldots,\left(j_{2 k}, h_{2 k}\right)}^{\left(j_{1}, h_{1}\right),\left(j_{2}, h_{2}\right), \ldots,\left(j_{k}, h_{k}\right)}\right)
\end{aligned}
$$

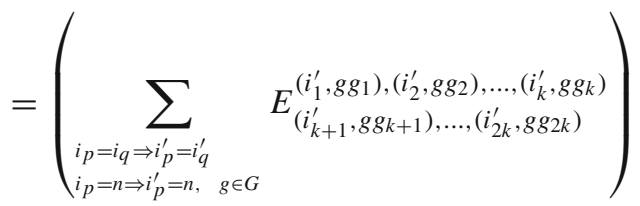

$$
\begin{aligned}
& \times\left(\sum_{\substack{j_{p}=j_{q} \Rightarrow j_{p}^{\prime}=j_{q}^{\prime} \\
j_{p}=n \Rightarrow j_{p}^{\prime}=n, h \in H}} E_{\left(j_{k+1}^{\prime}, h h_{k+1}\right), \ldots,\left(j_{2 k}, h h_{2 k}\right)}^{\left(j_{1}^{\prime}, h h_{1}\right),\left(j_{2}^{\prime}, h h_{2}\right), \ldots,\left(j_{k}^{\prime}, h h_{k}\right)}\right) \\
& =\sum E_{\left(i_{k+1}^{\prime}, g g_{k+1}\right),\left(i_{k+2}^{\prime}, g g_{k+2}\right), \ldots,\left(i_{2 k}^{\prime}, g g_{2 k}\right)}^{\left(i_{1}^{\prime}, g g_{1}\right),\left(i_{2}^{\prime}, g g_{2}\right), \ldots,\left(i_{k}^{\prime}, g g_{k}\right)} E_{\left(j_{k+1}^{\prime}, h h_{k+1}\right),\left(j_{k+2}^{\prime}, h h_{k+2}\right), \ldots,\left(j_{2 k}^{\prime}, h h_{2 k}\right)}^{\left(j_{1}^{\prime}, h h_{1}\right),\left(j_{2}^{\prime}, h h_{2}\right), \ldots,\left(j_{k}^{\prime}, h h_{k}\right)} \\
& =\sum \delta_{\left(j_{k+1}^{\prime}, h h_{k+1}\right),\left(j_{k+2}^{\prime}, h h_{k+2}\right), \ldots,\left(j_{2 k}^{\prime}, h h_{2 k}\right)}^{\left(i_{1}^{\prime}, g g_{1}\right),\left(i_{2}^{\prime}, g g_{2}\right), \ldots,\left(i_{k}^{\prime}, g g_{k}\right)} E_{\left(i_{k+1}^{\prime}, g g_{k+1}\right),\left(i_{k+2}^{\prime}, g g_{k+2}\right), \ldots,\left(i_{2 k}^{\prime}, g g_{2 k}\right)}^{\left(j_{1}^{\prime}, h h_{1}\right),\left(j_{2}^{\prime}, h h_{2}\right), \ldots,\left(j_{k}^{\prime}, h h_{k}\right)},
\end{aligned}
$$

(since $E_{p q} E_{r s}=\delta_{q r} E_{p s}$, where $\delta_{q r}$ is the Kronecker delta)

$=0$ if and only if $g\left(g_{1}, g_{2}, \ldots, g_{k}\right) \neq\left(h_{k+1}, h_{k+2}, \ldots, h_{2 k}\right)$ for some $g \in G$.

(b)

$$
\left(\widetilde{L}_{\left(i_{k+1}, g_{k+1}\right),\left(i_{k+2}, g_{k+2}\right), \ldots,\left(i_{2 k}, g_{2 k}\right)}^{\left(i_{1}, g_{1}\right),\left(i_{2}, g_{2}\right), \ldots,\left(i_{k}, g_{k}\right)}\right)\left(\widetilde{L}_{\left(j_{k+1}, h_{k+1}\right),\left(j_{k+2}, h_{k+2}\right), \ldots,\left(j_{2 k}, h_{2 k}\right)}^{\left(j_{1}, h_{1}\right),\left(j_{2}, h_{2}\right), \ldots,\left(j_{k}, h_{k}\right)}\right)
$$




$$
\begin{aligned}
& =\left(\sum_{\substack{i_{p}=i_{q} \Rightarrow i_{p}^{\prime}=i_{q}^{\prime} \\
i_{p}=n \Rightarrow i_{p}^{\prime}=n}} E_{\left(i_{k+1}^{\prime}, g_{k+1}\right), \ldots,\left(i_{2 k}^{\prime}, g_{2 k}\right)}^{\left(i_{1}^{\prime}, g_{1}\right),\left(i_{2}^{\prime}, g_{2}\right), \ldots,\left(i_{k}^{\prime}, g_{k}\right)}\right)\left(\sum_{\substack{j_{p}=j_{q} \Rightarrow j_{p}^{\prime}=j_{q}^{\prime} \\
j_{p}=n \Rightarrow j_{p}^{\prime}=n}} E_{\left(j_{k+1}^{\prime}, h_{k+1}\right), \ldots,\left(j_{2 k}, h_{2 k}\right)}^{\left(j_{1}^{\prime}, h_{1}\right),\left(j_{2}^{\prime}, h_{2}\right), \ldots,\left(j_{k}^{\prime}, h_{k}\right)}\right) \\
& =\sum E_{\left(i_{k+1}^{\prime}, g_{k+1}\right),\left(i_{k+2}^{\prime}, g_{k+2}\right), \ldots,\left(i_{2 k}^{\prime}, g_{2 k}\right)}^{\left(i_{1}^{\prime}, g_{1}\right),\left(i_{2}^{\prime}, g_{2}\right), \ldots,\left(i_{k}^{\prime}, g_{k}\right)} E_{\left(j_{k+1}^{\prime}, h_{k+1}\right),\left(j_{k+2}^{\prime}, h_{k+2}\right), \ldots,\left(j_{2 k}^{\prime}, h_{2 k}\right)}^{\left(j_{1}^{\prime}, h_{1}\right),\left(j_{2}^{\prime}, h_{2}\right), \ldots,\left(j_{k}^{\prime}, h_{k}\right)} \\
& =\sum \delta_{\left(j_{k+1}^{\prime}, h_{k+1}\right),\left(j_{k+2}^{\prime}, h_{k+2}\right), \ldots,\left(j_{2 k}^{\prime}, h_{2 k}\right)}^{\left(i_{1}^{\prime}, g_{1}\right),\left(i_{2}^{\prime}, g_{2}\right), \ldots,\left(i_{k}^{\prime}, g_{k}\right)} E_{\left(i_{k+1}^{\prime}, g_{k+1}\right),\left(i_{k+2}^{\prime}, g_{k+2}\right), \ldots,\left(i_{2 k}^{\prime}, g_{2 k}\right)}^{\left(j_{1}^{\prime}, h_{1}\right),\left(j_{2}^{\prime}, h_{2}\right), \ldots,\left(j_{k}^{\prime}, h_{k}\right)} \\
& =0 \text { if and only if }\left(g_{1}, g_{2}, \ldots, g_{k}\right) \neq\left(h_{k+1}, h_{k+2}, \ldots, h_{2 k}\right) \text {. }
\end{aligned}
$$

Lemma 3.3.2 (a) For each $g \in G$,

$$
\begin{aligned}
& \left(L_{\left(i_{k+1}, g_{k+1}\right),\left(i_{k+2}, g_{k+2}\right), \ldots,\left(i_{2 k}, g_{2 k}\right)}^{\left(i_{1}, g_{1}\right),\left(i_{2}, g_{2}\right), \ldots,\left(i_{k}, g_{k}\right)}\right)\left(L_{\left(j_{k+1}, g g_{1}\right),\left(j_{k+2}, g g_{2}\right), \ldots,\left(j_{2 k}, g g_{k}\right)}^{\left(j_{1}, h_{1}\right),\left(j_{2}, h_{2}\right), \ldots,\left(j_{k}, h_{k}\right)}\right) \\
& =(n)^{\lambda} L_{\left(s_{k+1}, g g_{k+1}\right),\left(s_{k+2}, g g_{k+2}\right), \ldots,\left(s_{2 k}, g g_{2 k}\right)}^{\left(s_{1}, h_{1}\right),\left(s_{2}, h_{2}\right), \ldots,\left(s_{k}, h_{k}\right)},
\end{aligned}
$$

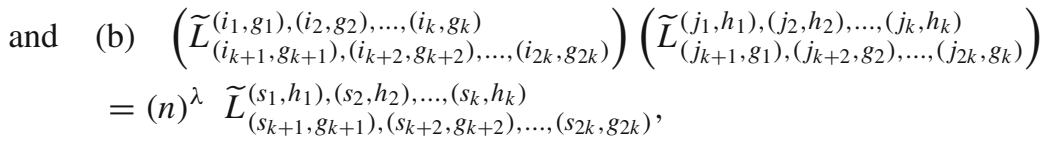

where $\lambda$ is the number of middle components in the product $d_{N} d_{N^{\prime}}^{\prime}$ (where $d_{N}$ and $d_{N^{\prime}}^{\prime}$ are defined in Lemma 3.2.2) and $\left(1 \leq s_{1}, s_{2}, \ldots, s_{2 k} \leq n\right)$ such that $p \sim q$ in $d_{N} d_{N^{\prime}}^{\prime} \Leftrightarrow s_{p}=s_{q}$.

Proof (a) For each $g \in G$

$$
\begin{aligned}
& \left(L_{\left(i_{k+1}, g_{k+1}\right),\left(i_{k+2}, g_{k+2}\right), \ldots,\left(i_{2 k}, g_{2 k}\right)}^{\left(i_{1}, g_{1}\right),\left(i_{2}, g_{2}\right), \ldots,\left(i_{k}, g_{k}\right)}\right)\left(L_{\left(j_{k+1}, g g_{1}\right),\left(j_{k+2}, g g_{2}\right), \ldots,\left(j_{2 k}, g g_{k}\right)}^{\left(j_{1}, h_{1}\right),\left(j_{2}, h_{2}\right), \ldots,\left(j_{k}, h_{k}\right)}\right) \\
& =\left(\sum_{\substack{i_{p}=i_{q} \Rightarrow i_{p}^{\prime}=i_{q}^{\prime} \\
i_{p}=n \Rightarrow i_{p}^{\prime}=n, g^{\prime} \in G}} E_{\left(i_{k+1}^{\prime}, g^{\prime} g_{k+1}\right), \ldots,\left(i_{2 k}^{\prime}, g^{\prime} g_{2 k}\right)}^{\left(i_{1}^{\prime}, g^{\prime} g_{1}\right),\left(i_{2}^{\prime}, g^{\prime} g_{2}\right), \ldots,\left(i_{k}^{\prime}, g^{\prime} g_{k}\right)}\right) \\
& \times\left(\sum_{\substack{j_{p}=j_{q} \Rightarrow j_{p}^{\prime}=j_{q}^{\prime} \\
j p=n \Rightarrow j_{p}^{\prime}=n, h^{\prime} \in G}} E_{\left(j_{k+1}^{\prime}, h^{\prime} g_{1}\right), \ldots,\left(j_{2 k}^{\prime}, h^{\prime} g_{2 k}\right)}^{\left(j_{1}^{\prime}, h^{\prime} h_{1}\right),\left(j_{2}^{\prime}, h^{\prime} h_{2}\right), \ldots,\left(j_{k}^{\prime}, h^{\prime} h_{k}\right)}\right) \\
& =\sum E_{\left(i_{k+1}^{\prime}, g^{\prime} g_{k+1}\right), \ldots,\left(i_{2 k}^{\prime}, g^{\prime} g_{2 k}\right)}^{\left(i_{1}^{\prime}, g^{\prime} g_{1}\right),\left(i_{2}^{\prime}, g^{\prime} g_{2}\right), \ldots,\left(i_{k}^{\prime}, g^{\prime} g_{k}\right)} \sum E_{\left(j_{k+1}^{\prime}, h^{\prime} g_{1}\right), \ldots,\left(j_{2 k}^{\prime}, h^{\prime} g_{2 k}\right)}^{\left(j_{1}^{\prime}, h^{\prime} h_{1}\right),\left(j_{2}^{\prime}, h^{\prime} h_{2}\right), \ldots,\left(j_{k}^{\prime}, h^{\prime} h_{k}\right)}
\end{aligned}
$$




$$
\begin{aligned}
& =\sum \delta_{\left(i_{1}^{\prime}, g g_{1}\right),\left(i_{2}^{\prime}, g g_{2}\right), \ldots,\left(i_{k}^{\prime}, g g_{k}\right)}^{\left(j_{k+1}^{\prime}, h g^{\prime} g_{1}\right),\left(j_{k+1}^{\prime}, h g^{\prime} g_{2}\right), \ldots,\left(j_{2 k}^{\prime}, h g^{\prime} g_{2 k}\right)} E_{\left(i_{k+1}^{\prime}, g g_{k+1}\right),\left(i_{k+2}^{\prime}, g g_{k+2}\right), \ldots,\left(i_{2 k}^{\prime}, g g_{2 k}\right)}^{\left(j_{1}^{\prime}, h h_{1}\right),\left(j^{\prime}, h h_{2}\right), \ldots,\left(j_{k}^{\prime}, h h_{k}\right)} \\
& =\sum \delta_{\left(i_{1}^{\prime}, i_{2}^{\prime}, \ldots, i_{k}^{\prime}\right)}^{\left(j_{k+1}^{\prime}, j_{k+2}^{\prime}, \ldots, j_{2 k}^{\prime}\right)} E_{\left(i_{k+1}^{\prime}, h g^{\prime} g_{k+1}\right),\left(i_{k+2}^{\prime}, h g^{\prime} g_{k+2}\right), \ldots,\left(i_{2 k}^{\prime}, h g^{\prime} g_{2 k}\right)^{\prime}}^{\left(j_{1}^{\prime}, h h_{1}\right),\left(j_{2}^{\prime}, h h_{2}\right), \ldots,\left(j_{k}^{\prime}, h h_{k}\right)}
\end{aligned}
$$

The number of times $E_{\left(i_{k+1}^{\prime}, h g^{\prime} g_{k+1}\right),\left(i_{k+2}^{\prime}, h g^{\prime} g_{k+2}\right), \ldots,\left(i_{2 k}^{\prime}, h g^{\prime} g_{2 k}\right)}^{\left(j^{\prime}, h h_{1}\right),\left(j^{\prime}, h h_{2}\right), \ldots,\left(j^{\prime}, h h_{k}\right)}$ appears in the above sum is equal to the number of pairs of sequences

$$
\left\{\begin{array}{l}
j_{k+1}^{\prime}, j_{k+2}^{\prime}, \ldots, j_{2 k}^{\prime}, j_{1}^{\prime}, j_{2}^{\prime}, \ldots, \\
i_{1}^{\prime}, \quad i_{2}^{\prime}, \ldots, i_{k}^{\prime}, \quad i_{k+1}^{\prime}, i_{k+2}^{\prime}, \ldots, i_{2 k}^{\prime}
\end{array}\right.
$$

such that

(i) $i_{p}=i_{q} \Rightarrow i_{p}^{\prime}=i_{q}^{\prime}$ and $i_{p}=n \Rightarrow i_{p}^{\prime}=n$

(ii) $j_{p}=j_{q} \Rightarrow j_{p}^{\prime}=j_{q}^{\prime}$ and $j_{p}=n \Rightarrow j_{p}^{\prime}=n$

(iii) $i_{1}^{\prime}, i_{2}^{\prime}, \ldots, i_{k}^{\prime}$ and $j_{k+1}^{\prime}, j_{k+2}^{\prime}, \ldots, j_{2 k}^{\prime}$ are fixed.

(iv) $i_{k+1}^{\prime}=j_{1}^{\prime}, i_{k+2}^{\prime}=j_{2}^{\prime}, \ldots, i_{2 k}^{\prime}=j_{k}^{\prime}$

Suppose there is a middle component in the product $d_{N} d_{N^{\prime}}^{\prime}$ then we can give $n$ values for the corresponding component in Eq. (3.14) to get different sequence. Hence (3.13) can be written as in (3.14). This comes from a sequence of purely combinatorial arguments as in the case of the partition algebra. Proof of (b) is similar to the proof of (a).

Theorem 3.3.3 (a) The map $\widehat{\phi}: \widehat{P}_{k+\frac{1}{2}}(n, G) \longrightarrow$ End $\left(W^{\otimes k}\right)$ is an algebra homomorphism onto on End $_{S_{n-1}} W^{\otimes k}$.

(b) The restricted map $\phi: P_{k+\frac{1}{2}}(n, G) \longrightarrow E n d\left(W^{\otimes k}\right)$ is onto on $E n d_{G \times S_{n-1}} W^{\otimes k}$.

Proof (a) It is enough to prove $\widehat{\phi}\left\{\left(d_{N}, f\right) *\left(d_{N^{\prime}}^{\prime}, f^{\prime}\right)\right\}=\widehat{\phi}\left(d_{N}, f\right) \widehat{\phi}\left(d_{N^{\prime}}, f^{\prime}\right)$, where $\left(d_{N}, f\right),\left(d_{N^{\prime}}, f^{\prime}\right)$ are $\left(G, k+\frac{1}{2}\right)$-diagrams. So the homomorphism is an immediate consequence of Lemma 3.3.1 and Lemma 3.3.2. Note that each $\widetilde{L}_{J}^{I}$ has pre image such that $\widehat{\phi}\left(d\left(i_{1}, i_{2}, \ldots, i_{2 k}\right)_{N}, f\right)=\widetilde{L}_{\left(i_{k+1}, f(k+1),\left(i_{k+2}, f(k+2), \ldots,\left(i_{2 k}, f(2 k)\right.\right.\right.}^{\left(i_{1}, f(1),\left(i_{2}, f(2), \ldots,\left(i_{k}, f(k)\right.\right.\right.}$, where $N=\left\{p \in[2 k] \mid i_{p}=n\right\}$.

(b) From Lemma 3.2.1, We have

$$
\begin{aligned}
\widehat{\phi}\left(\widehat{d_{N}, f}\right) & =\widehat{\phi}\left(\sum_{\bar{g} \in \bar{G}}\left(d_{N}, \bar{g} f\right)\right) \\
& =\sum_{\bar{g} \in \bar{G}} \widehat{\phi}\left(d_{N}, \bar{g} f\right)
\end{aligned}
$$




$$
\begin{aligned}
& =\sum_{g \in G} \sum_{\substack{x \sim y \text { in } d \Rightarrow j_{x}=j_{y} \\
x \in N \Rightarrow i_{x}=n}} E_{\left(j_{k+1}, g f(k+1)\right),\left(j_{k+2}, g f(k+2)\right), \ldots,\left(j_{2 k}, g f(2 k)\right)}^{\left(j_{1}, g f(1)\right),\left(j_{2}, g f(2)\right), \ldots,\left(j_{k}, g f(k)\right)} \\
& =\sum_{\substack{x \sim y \text { in } \\
g \in G, x \in N \Rightarrow j_{x}=j_{y} \\
g \in i_{x}=n}} E_{\left(j_{k+1}, g f(k+1)\right),\left(j_{k+2}, g f(k+2)\right), \ldots,\left(j_{2 k}, g f(2 k)\right)}^{\left(j_{1}, g f(1)\right),\left(j_{2}, g f(2)\right), \ldots,\left(j_{k}, g f(k)\right)} \text {. }
\end{aligned}
$$

Thus the matrices $\widehat{\phi}\left(\widehat{d_{N}, f}\right) \in E n d_{G \times s_{n-1}}$ (Using (3.4)). Note that each $L_{J}^{I}$ has pre image such that $\widehat{\phi}\left(d\left(i_{1}, i_{2}, \ldots, i_{2 k}\right)_{N}, f\right)=L_{\left(i_{k+1}, f(k+1),\left(i_{k+2}, f(k+2), \ldots,\left(i_{2 k}, f(2 k)\right.\right.\right.}^{\left(i_{1}, f(1),\left(i_{2}, f(2), \ldots,\left(i_{k}, f(k)\right.\right.\right.}$, where $N=\left\{p \in[2 k] \mid i_{p}=n\right\}$.

Corollary 3.3.4 $S_{n-1}$ and $\widehat{P}_{k+\frac{1}{2}}(n, G)$ generate full centralizers of each other in $\operatorname{End}\left(W^{\otimes k}\right)$. In particular, for $n-1 \geq 2 k$, we have (a) $\widehat{P}_{k+\frac{1}{2}}(n, G) \cong \operatorname{End}_{S_{n-1}}\left(W^{\otimes k}\right)$, (b) $\mathbb{C}\left(S_{n-1}\right)$ generates End $\widehat{P}_{k+\frac{1}{2}}\left(W^{\otimes k}\right)$.

Proof The proof is the immediate consequence of Theorem 3.3.3. Since $n-1 \geq$ $2 k, \operatorname{dim} \widehat{P}_{k+\frac{1}{2}}(n, G)=\operatorname{dim} \operatorname{End}_{S_{n-1}}(W \otimes k)$. In the proof of Theorem 3.3.3, we have $\widehat{\phi}\left(\widehat{P}_{k+\frac{1}{2}}(n, G)\right) \subseteq \operatorname{End}_{S_{n-1}}\left(W^{\otimes k}\right)$. As $\left(d_{N}, f\right)$ ranges over all diagrams, all $\widehat{L}_{d}$ are obtained. Thus the representation $\widehat{\phi}$ takes a basis of $\widehat{P}_{k+\frac{1}{2}}(n, G)$ to a basis of $E n d_{S_{n-1}}\left(W^{\otimes k}\right)$, so $\widehat{P}_{k+\frac{1}{2}}(n, G) \cong E n d_{S_{n-1}}\left(W^{\otimes k}\right)$. Proof of (b): This follows from (a) and the double centralizer Theorem.

Corollary 3.3.5 $G \times S_{n-1}$ and $P_{k+\frac{1}{2}}(n, G)$ generate full centralizers of each other in End $\left(W^{\otimes k}\right)$. In particular, for $n-1 \geq 2 k$, we have (a) $P_{k+\frac{1}{2}}(n, G) \cong \operatorname{End}_{G \times S_{n-1}}$ $\left(W^{\otimes k}\right)$, (b) $\mathbb{C}\left(G \times S_{n-1}\right)$ generates End $_{P_{k+\frac{1}{2}}}\left(W^{\otimes k}\right)$.

Proof The proof is similar to the Corollary 3.3.3.

As centralizers of the semisimple group algebras $\mathbb{C}\left(S_{n-1}\right)$ and $\mathbb{C}\left(G \times S_{n-1}\right)$ respectively, the $\mathbb{C}$-algebras $\widehat{P}_{k+\frac{1}{2}}(n, G)$ and $P_{k+\frac{1}{2}}(n, G)$ are semisimple for $n-1 \geq 2 k$.

Open Access This article is distributed under the terms of the Creative Commons Attribution License which permits any use, distribution, and reproduction in any medium, provided the original author(s) and the source are credited.

\section{References}

1. Bloss, M.: $G$-colored partition algebras as centralizer algebras of wreath products. J. Algebra $\mathbf{2 6 5}$, 690-710 (2003)

2. Grood, C.: The rook partition algebra. J. Combin. Theory Ser. A 113, 325-351 (2006)

3. Halverson, T., Ram, A.: The Partition algebras. Eur. J. Combin. 26, 869-921 (2005)

4. Halverson, T., Lewandowski, T.: RSK insertion for set partitions and diagram algebras. Electron. J. Combin. 11(2), R24 (2004)

5. Jones, V.F.R.: The Potts model and the symmetric group. In: Subfactors: Proceedings of the Taniguchi Symposium on Operater Algebra, Kyuzeso, 1993, pp. 259-267. World Scientific, River edge (1994)

6. Joseph Kennedy, A.: Class partition algebras as centralizer algebras of wreath products. Commun. Algebra 35, 145-170 (2007) 
7. Martin, P.: Temperley Lieb algebras for non planar statistical mechanics: the partition algebra construction. J. Knot Theory Ramifications 3, 51-82 (1994)

8. Martin, P.P., Elgamal, A.: Ramified partition algebras. Math. Z. 246, 473-500 (2004)

9. Martin, P., Rollett, G.: The Potts model representation and a Robinson-Schensted correspondence for the partition algebra. Compos. Math. 112, 237-254 (1998)

10. Martin, P., Saleur, H.: On an algebraic approach to higher-dimensional statistical mechanics. Commun. Math. Phys. 158, 155-190 (1993)

11. Parvathi, M., Joseph Kennedy, A.: $G$-vertex colored partition algebras as centralizer algebras of direct products. Commun. Algebra 32(11), 4337-4361 (2004)

12. Parvathi, M., Joseph Kennedy, A.: Extended $G$-vertex colored partition algebras as centralizer algebras of symmetric groups. J. Algebra Discrete Math. 2, 46-67 (2005)

13. Parvathi, M., Joseph Kennedy, A.: Representations of Vertex Colored Partition Algebras. Southeast Asian Bull. Math. 28, 493-518 (2004) 